\title{
Pixel Telescope to test pixel Phase II ROCs and sensors
}

Caleb Fangmeier*

Univ. of Nebraska - Lincoln

E-mail: cfangmeidcern.ch

\begin{abstract}
A silicon-strip sensor based particle telescope is being developed to aid in the characterization of next-generation silicon-pixel detectors for the Compact Muon Solenoid (CMS) detector at the Large Hadron Collider (LHC). This telescope is expected to enable sub-micron feature studies of prototype detectors by combining $25-\mu \mathrm{m}$ pitch strip detectors with a very low noise readout system. Additionally, this telescope improves upon previous iterations by deploying a parallel readout scheme to increase the maximum trigger rate by a factor of 16 .
\end{abstract}

38th International Conference on High Energy Physics 3-10 August 2016

Chicago, USA

${ }^{*}$ Speaker. 


\section{Introduction}

The innermost element of the CMS detector at the LHC is the silicon pixel tracker. The current version of the tracker has performed well and been critical to the physics program of CMS. The High Luminosity LHC upgrade of CMS will replace the entire silicon tracking system. As part of this upgrade, a new section of the pixel tracker is proposed to be added in the so-called "very-forward" $(\eta \sim 4)$ region of the detector. Because the particles in this region are traveling almost parallel to the magnetic field of the detector, enhanced $\phi$ sensitivity is required to accurately measure track curvature, a critical measurement for finding particle momentum and charge. The proposed new detector section will feature pixels with increased elongation, relative to the current CMS pixel tracker, for enhanced $\phi$ sensitivity, while sacrificing sensitivity in $\rho$. This contribution presents a telescope being developed primarily to characterize these elongated pixel geometries.

The telescope consists of eight layers of silicon-strip sensors, with the prototype pixel detector placed in the center surrounded by four strip sensor layers on either side. A beam of charged particles is directed to pass through both the strip sensors and the prototype pixel sensors. Measurements from the telescope are taken to reconstruct individual particle tracks. These tracks are then compared to data collected from the pixel sensor to characterize its performance.

\section{Hardware}

The telescope has been designed around a specific silicon-strip sensor and accompanying readout chip, both of which were originally developed for the H1 vertex detector at HERA [1]. The strip sensor contains 512 strips with $25 \mu \mathrm{m}$ pitch. The readout chip is the "Analog Pipeline Chip 128" (APC). Each APC can read 128 strips so four chips are required to read a single strip sensor. The APC operates an integrating pre-amplifier that collects charge from a single sensor strip and keeps a 32-sample history of the output of that pre-amplifier. When a trigger is received from, for example, a scintillation detector indicating a particle has passed through the telescope, the appropriate sample is chosen from the 32-sample history based on the calibrated trigger latency and the selected samples from all 128 channels of the APC are serialized to its output.

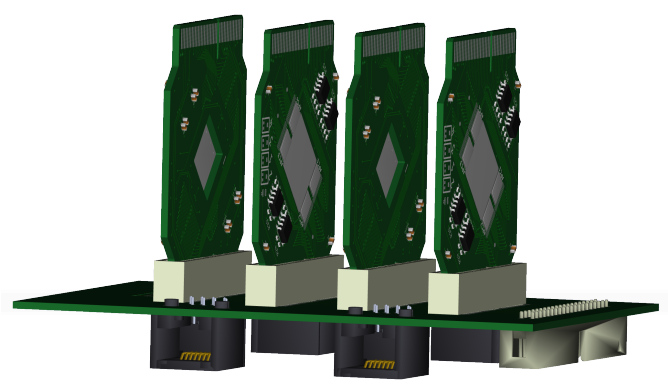

Fig. 1.a: A rendering of the telescope "motherboard" showing four "sensor-cards" each of which holds a single strip sensor. The control signals needed by the readout chip are supplied by a 40-pin header (bottom-right). The data readout happens over four RJ-45 connectors (bottom-center), into which CAT-5 cables are plugged to transmit the signals to the DAQ board.

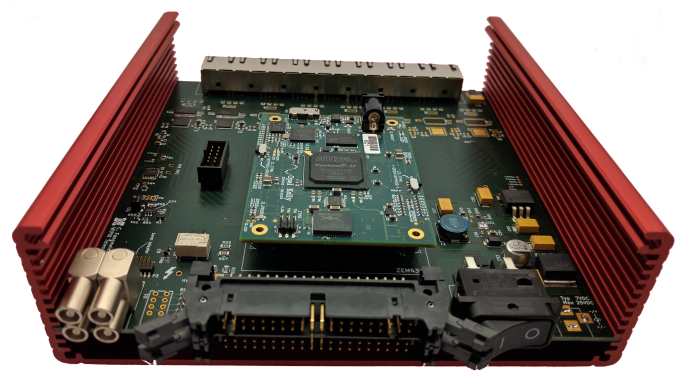

Fig. 1.b: An image of the DAQ board featuring an $8 \times$ RJ -45 connector (back) to receive data from two connected motherboards, a $2 \times 40$-pin header (front) for supplying control signals to the motherboards, and an Opal-Kelly FPGA integration module (center) to orchestrate the operation of the telescope. 
The strip sensor and four APC are mounted onto a sensor-card along with four buffer amplifiers that compensate for the relatively weak output of the APC and generate a more robust differential signal with the proper output impedance for driving CAT-5 cables $(100 \Omega)$. Four sensor-cards are plugged into a motherboard (Fig. 1.a) which provides mechanical support for the sensor-cards and routes electrical signals between the sensor cards and the I/O ports.

The signals from the two motherboards that constitute the telescope are routed to the data acquisition (DAQ) board (Fig. 1.b). The DAQ board contains eight 40MHz 10-bit ADCs with four channels each, giving a total of 32 digitization channels. The DAQ board also contains circuitry for supplying the bias voltage for the strip detectors, and LEMO connectors for supplying an external trigger and clock signal. Digital logic is implemented via an Opal Kelly FPGA integration module which features an Altera Cyclone IV FPGA, 128MB of RAM, and a Super-Speed USB link for data transfer.

\section{Readout Scheme}

The readout scheme has been designed to minimize the time required to transfer the strip sensor pulse-height values from the APC to the DAQ board. The APC cannot record data during readout so increasing the data transfer speed results in a reduction of dead time. As illustrated in Fig. 2, the readout happens in three stages.

The first stage is handled by the electronics in and near the beam. It is here that the ionization caused by the passage of a charged particle is converted to an electronic pulse by the strip sensor and the height of that pulse is recorded by the APC. The APC then serializes pulse heights from many strips to the cables exiting the beam region.

In the second stage, signals from the telescope are received by the DAQ board where they are digitized and fed into an FPGA. The FPGA contains firmware that is responsible for dropping strip readings with pulse-heights below a set threshold and grouping the remaining strips into "hit" objects. These hit objects, along with their associated trigger id, are then pushed to a connected PC via a USB link.

The third stage consists of software that receives the hit data from the DAQ board, constructs events from them, and saves the events to disk. Offline analysis software then uses this data to identify the individual tracks of particles passing through the telescope. Detector alignment studies will also be critical to measuring the position of the strip sensors to micron precision. Finally, the tracks can be interpolated to the impact point with the prototype pixel detector and used to measure its properties.

\section{Projected Telescope Performance}

The performance of the telescope can be quantified in two ways: the precision with which individual particle trajectories can be measured, and the rate at which data can be collected.

The measurement precision of particle tracks depends on the number of detector layers and the single layer spatial resolution. The telescope consists of eight silicon-strip sensor layers with $25 \mu \mathrm{m}$ pitch. For strip sensors with charge sharing the single hit precision can be approximated by the pitch divided by the signal-to-noise ratio of the readout system. The expected signal-to-noise ratio 


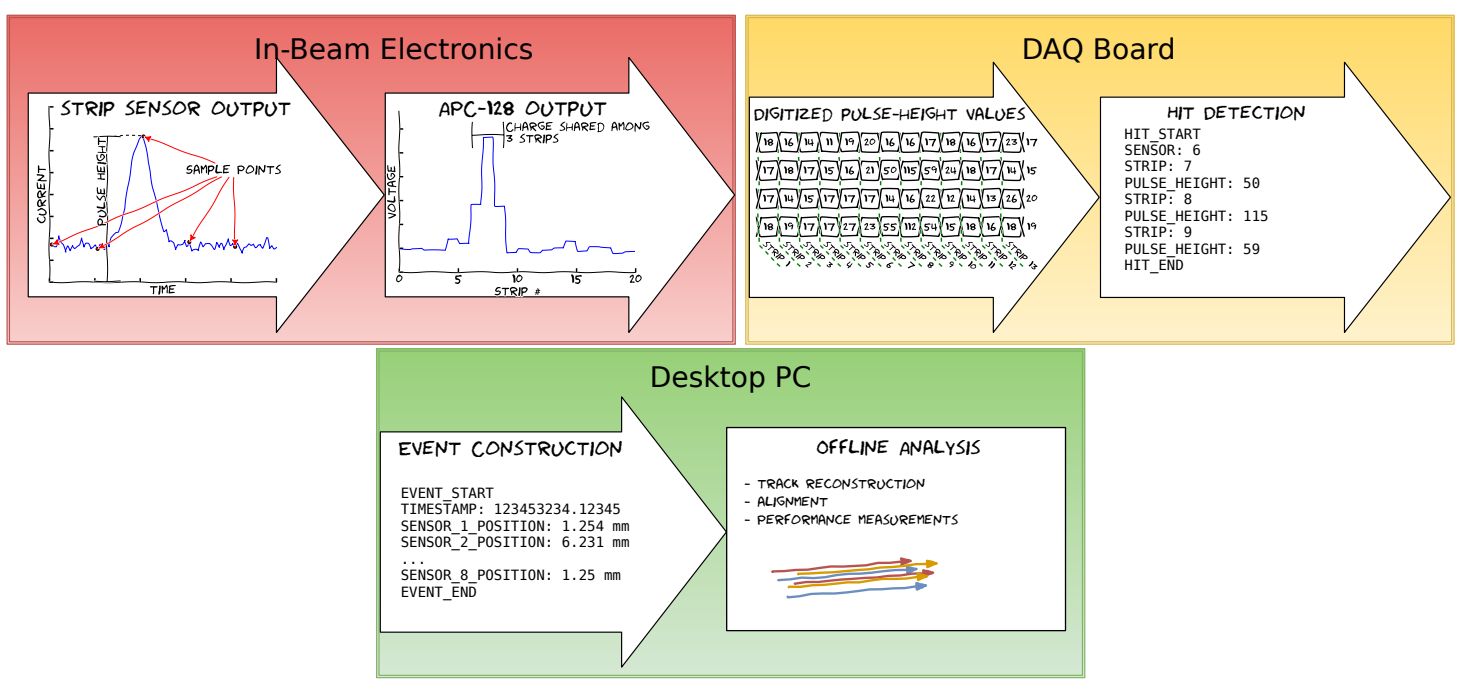

Figure 2: Illustration of the readout scheme of the telescope.

of the telescope is 25 [2]. This yields a single-hit precision of $\approx 1 \mu \mathrm{m}$. This single-hit precision combined with two measurements in each dimension on each side of the prototype sensor should give sub-micron track localization at the point of impact with the prototype sensor.

Because many test-beam facilities have beam rates on the order of $\mathrm{MHz}$, only a small fraction of time during data taking will be spent waiting for a trigger. Therefore, increasing the telescope's maximum trigger rate will significantly increase the amount of data that can be collected during a test beam. Furthermore, the trigger rate for the telescope is primarily limited by the time required to transfer 128 pulse-height values from the APC to the DAQ Board. A previous version of this telescope used the daisy-chain feature of the APC to serialize the readout of 16 APC into a single readout channel[3]. This simplified the DAQ because it reduced the number of digitization channels from 32 to 2, but also increased the readout time by a factor of 16 . The new version of the telescope will avoid this performance penalty by reading out all 32 APC in parallel. In addition to parallel readout, the trigger rate can be improved by optimizing the readout electronics using well established RF design techniques. For example, placing buffer amplifiers near the APC will reduce the rise-time of signals as they propagate along the cables to the DAQ board, while using differential signaling on these same cables will reduce electro-magnetic interference from the environment. With these improvements a maximum trigger rate of $\approx 15 \mathrm{kHz}$ is expected.

\section{References}

[1] M. Hilgers, Development of a radiation hard version of the Analog Pipeline Chip APC128, Nucl. Instrum. Meth. A481:556-565,2002 [hep-ex/0101023].

[2] A. Ryser (2013). Pixel Telescope (Report, ETH Zurich).

[3] P. Turner (2012). Design and Implementation of a High Resolution Telescope for CMS Pixel Detector Characterization, (Report, Univ. of Illinois at Chicago). 